\title{
Ralstonia solanacearum Depends on Catabolism of Myo-Inositol, Sucrose, and Trehalose for Virulence in an Infection Stage-Dependent Manner
}

\author{
Corri D. Hamilton, ${ }^{1}$ Olivia R. Steidl, ${ }^{1}$ April M. MacIntyre, ${ }^{1,2}$ Connor G. Hendrich, ${ }^{1,2}$ and Caitilyn Allen ${ }^{1, \dagger}$ \\ ${ }^{1}$ Department of Plant Pathology University of Wisconsin-Madison, 1630 Linden Drive, Madison, WI 53706, U.S.A. \\ ${ }^{2}$ Microbiology Doctoral Training Program, University of Wisconsin-Madison, 1630 Linden Drive, Madison, WI 53706, U.S.A. \\ Accepted 20 January 2021.
}

The soilborne pathogen Ralstonia solanacearum causes a lethal bacterial wilt disease of tomato and many other crops by infecting host roots, then colonizing the water-transporting xylem vessels. Tomato xylem sap is nutritionally limiting but it does contain some carbon sources, including sucrose, trehalose, and myo-inositol. Transcriptomic analyses revealed that $\boldsymbol{R}$. solanacearum expresses distinct catabolic pathways at low cell density (LCD) and high cell density (HCD). To investigate the links between bacterial catabolism, infection stage, and virulence, we measured in planta fitness of bacterial mutants lacking specific carbon catabolic pathways expressed at either LCD or HCD. We hypothesized that early in disease, during root infection, the bacterium depends on carbon sources catabolized at LCD, while HCD carbon sources are only required later in disease during stem colonization. A $R$. solanacearum $\Delta i o l G$ mutant unable to use the LCD-catabolized nutrient myo-inositol was defective in tomato root colonization, but after it reached the stem this strain colonized and caused symptoms as well as wild type. In contrast, $R$. solanacearum mutants unable to use the HCD-catabolized nutrients sucrose $(\Delta s c r A)$, trehalose $(\Delta t r e A)$, or both $(\Delta s c r A /$ tre $A)$, infected roots as well as wild-type $R$. solanacearum but were defective in colonization and competitive fitness in midstems and had reduced virulence. Further, xylem sap from tomato plants colonized by $\triangle s c r A, \Delta$ tre $A$, or $\triangle s c r A /$ treA $R$. solanacearum mutants contained twice as much sucrose as sap from plants colonized by wild-type $R$. solanacearum. Together, these findings suggest that quorum sensing specifically adapts $R$. solanacearum metabolism for success in the different nutritional environments of plant roots and xylem sap.

${ }^{\dagger}$ Corresponding author: C. Allen; callen@wisc.edu

Funding: This research was supported by the University of WisconsinMadison College of Agricultural and Life Sciences through a UWMadison Sophomore Research Award to O. R. Steidl, a National Science Foundation Predoctoral Fellowship to C. G. Hendrich, a UW-Madison SciMed-Graduate Research Scholars Fellowship to C. D. Hamilton, and a United States Department of Agriculture AFRI Predoctoral Fellowship to C. D. Hamilton and A. M. MacIntyre.

*The $\boldsymbol{e}$-Xtra logo stands for "electronic extra" and indicates there is supplementary material published online.

The author(s) declare no conflict of interest.
Keywords: bacterial catabolism, bacterial wilt disease, metabolomics, metabolic trade-offs, myo-inositol, sucrose, trehalose, xylem sap

Microbial success hinges on the ability to reduce the organic compounds available in a specific habitat. Microbes occupying niches with diverse but scarce nutrients need a broad set of catabolic pathways, but operating many such pathways imposes a cost (Dekel and Alon 2005; Fatima and Senthil-Kumar 2015; Gudelj et al. 2007; Pfeiffer and Bonhoeffer 2004; Polz and Cordero et al. 2016; Siebrecht et al. 2003). Sensing and signaling mechanisms help microbes make informed choices about when to express a given pathway to balance the energy cost of synthesizing transporters and enzymes with the energy gained from consuming the corresponding carbon sources (Wadhams and Armitage 2004).

The plant products myo-inositol, sucrose, and trehalose are potential energy sources for plant-associated microbes. Myo-inositol, a sugar alcohol, is often found in high quantities in planta. It is common in root exudates, including those of tomato; these exudates are the first plant nutrient interface for soil-borne microbes (Kravchenko et al. 2003; Wang and Bergeson 1974; Wei et al. 2015). Myo-inositol plays a vital role as the core of many signaling molecules and membrane and cell-wall structural components, and it is the most abundant carbon source in root nodules of the Bradyrhizobium diazoefficiens-pea mutualism (Sköt and Egsgaard 1984; Vílchez et al. 2020). Sucrose is the major product of photosynthesis. It is essential for plant growth and development, and plants continuously shuttle sucrose from source to sink (Pollock and Farrar 1996; Salanoubat and Belliard 1989; Sonnewald and Willmitzer 1992). The disaccharide trehalose, much less abundant than sucrose, plays key roles in plant metabolic regulation and stress tolerance (Lunn et al. 2014; Paul et al. 2008). Trehalose is a major contributor to tolerance of drought and temperature stress and plants use trehalose 6-phosphate signaling to balance their sucrose-to-starch storage ratio (Kolbe et al. 2005).

Ralstonia solanacearum is part of a heterogeneous species complex of plant pathogenic $\beta$-proteobacteria that cause bacterial wilt diseases (Allen et al. 2005; Elphinstone 2005; Genin and Denny 2012; Prior et al. 2016). Bacterial wilt affects over 100 economic crops, including tomato, potato, banana, tobacco, peanut, ginger, cloves, and eggplant, reducing yields for subsistence and commercial growers worldwide (Allen et al. 2005).

$R$. solanacearum cycles between two very different nutritional environments, rhizosphere soil and host xylem sap. The rhizosphere teems with diverse microbes and carbon sources (Yang and Crowley 2000). R. solanacearum is well adapted to this 
complex environment, with more than 50 catabolic pathways to use a wide variety of resources, although pathway repertoires vary somewhat within the species complex (Álvarez et al. 2010; Jacobs et al. 2012; Zuluaga et al. 2013). R. solanacearum is found at relatively low titers in bulk soil, reaching only $10^{4}$ CFU per gram in the most heavily infested fields (Allen et al. 2005; Hayward 1991).

Soil-dwelling $R$. solanacearum cells use chemotaxis to locate and infect tomato roots, moving to the developing vascular bundles and spreading systemically through the water transporting xylem (Caldwell et al. 2017; McGarvey et al. 1999). Xylem vessels are dead and xylem sap is described as a nutrient-poor solution of water and minerals (Evert and Eichhorn 2006). Nonetheless, $R$. solanacearum rapidly grows to cell densities $>10^{9}$ CFU per milliliter of xylem sap, eventually forming a biofilm matrix that can fill entire vessels and obstruct water flow (Denny 2006; Yao and Allen 2007). These high bacterial populations coincide with the onset of symptoms, which include stunting, leaf wilting, vascular discoloration, and death.

Little is understood about how $R$. solanacearum grows to such high cell densities in xylem sap. Xylem sap from healthy tomato plants contains enough sugars, amino acids, and organic acids to support only limited bacterial growth in vitro (Coplin et al. 1974; Dixon and Pegg 1972; Evert and Eichhorn 2006; Fatima and Senthil-Kumar 2015; Wang and Bergeson 1974; White et al. 1981). Interestingly, xylem sap from $R$. solanacearum-infected plants is enriched in certain metabolites, including carbon sources that can support $R$. solanacearum growth, such as sucrose and trehalose (Lowe-Power et al. 2018a). This metabolomic finding, together with the observation that $R$. solanacearum grows better in sap harvested from infected plants than in sap from healthy plants, suggests that the pathogen somehow increases available nutrients in xylem vessels.

When $R$. solanacearum grows to high cell densities (HCD) in culture or in the confined environment of the xylem vessel, the PhcA quorum-sensing (QS) system upregulates expression of secreted bacterial wilt virulence factors, like extracellular polymeric substances (EPS) and plant cell wall-degrading enzymes. Studies with a $\Delta p h c A$ mutant, which is genetically locked in a low cell density (LCD) state, revealed that this regulator also represses expression of dozens of transporters and catabolic pathways at HCD (Khokhani et al. 2017; Peyraud et al. 2016). In this way, $R$. solanacearum has evolved a metabolic trade-off between maximizing growth by using all available nutrients and maximizing pathogenic fitness by producing costly secreted virulence factors (Polz and Cordero 2016). In the context of the bacterial wilt disease cycle, this suggests that $R$. solanacearum uses a broad set of nutrients when it grows in the rhizosphere and during early root infection, but once the bacterium grows to large population sizes in xylem vessels, it shifts to catabolizing a much narrower set of compounds. These observations led us to hypothesize that $R$. solanacearum is adapted to use specific carbon sources at different points in its life history.
Here, we elucidate the relationships between $R$. solanacearum catabolism, infection stage, and ability to colonize and cause disease on tomato. We found that bacterial mutants lacking the ability to consume myo-inositol, sucrose, and trehalose had reduced fitness in an infection stage-specific manner. In particular, a LCD-induced gene encoding a myo-inositol-catabolizing enzyme was required only for infection, root endosphere colonization, and competitive fitness in roots very early in wilt disease development. In contrast, the HCD-induced genes encoding degradation of sucrose and trehalose were required for colonization and competitive fitness in the stem and for full virulence. Although sucrose is an important carbon source for $R$. solanacearum in the xylem, we found that the much less abundant sugar trehalose is not an important energy source for the bacterium in planta. However, trehalose degradation does contribute to $R$. solanacearum virulence by another mechanism.

\section{RESULTS}

\section{The catabolic genes iolG, scrA, and tre $A$ are expressed during tomato infection and are regulated by the PhcA QS system.}

$R$. solanacearum GMI1000 can grow on myo-inositol, sucrose, and trehalose as sole carbon sources, and all three metabolites are present in tomato xylem sap and root exudates (Lowe-Power et al. 2018a; Wang and Bergeson 1974; Wei et al. 2015). Infecting tomato plants with $R$. solanacearum increases levels in xylem sap of sucrose and myo-inositol less than twofold, but trehalose concentrations are 19-fold higher in $R$. solanacearum-infected sap than in sap from healthy plants (LowePower et al. 2018a). To determine the roles of these three carbon sources in the $R$. solanacearum life cycle, we mutated iolG, which encodes an oxidoreductase that is the first step in catabolism of myo-inositol, scrA, which encodes a sucrose-specific transport protein, and treA, which encodes an alpha-trehalase (Kanehisa and Goto 2000). As summarized in Table 1, during growth in tomato plants, $R$. solanacearum GMI1000 expresses iolG, scrA, and tre $A$ at the moderately high absolute expression levels of 10.15, 11.08, and 12.53, respectively (Jacobs et al. 2012). All three genes are regulated by the cell density-responsive PhcA QS system. While iolG is up-regulated in a phcA mutant, which is genetically locked in a LCD state, treA and $s c r A$ are both down-regulated in the $\Delta p h c A$ strain (Khokhani et al. 2017) (Table 1).

To determine the roles of these three carbon sources across the $R$. solanacearum infection process, we created $R$. solanacearum mutants, $\Delta i o l G, \Delta s c r A$, and $\Delta$ treA, lacking the three targeted catabolism pathways, respectively, and confirmed their phenotypes by measuring mutant growth on glucose, myo-inositol, sucrose, and trehalose as sole carbon sources (Supplementary Table S1). The $\Delta i o l G, \Delta s c r A, \Delta t r e A$, and $\Delta s c r A /$ treA double mutant all grew as well as wild-type strain GMI1000 on glucose. As expected, $\Delta i o l G, \Delta s c r A, \Delta$ treA, and $\Delta s c r A /$ treA differed from wild type only

Table 1. Ralstonia solanacearum catabolic genes and metabolites of interest

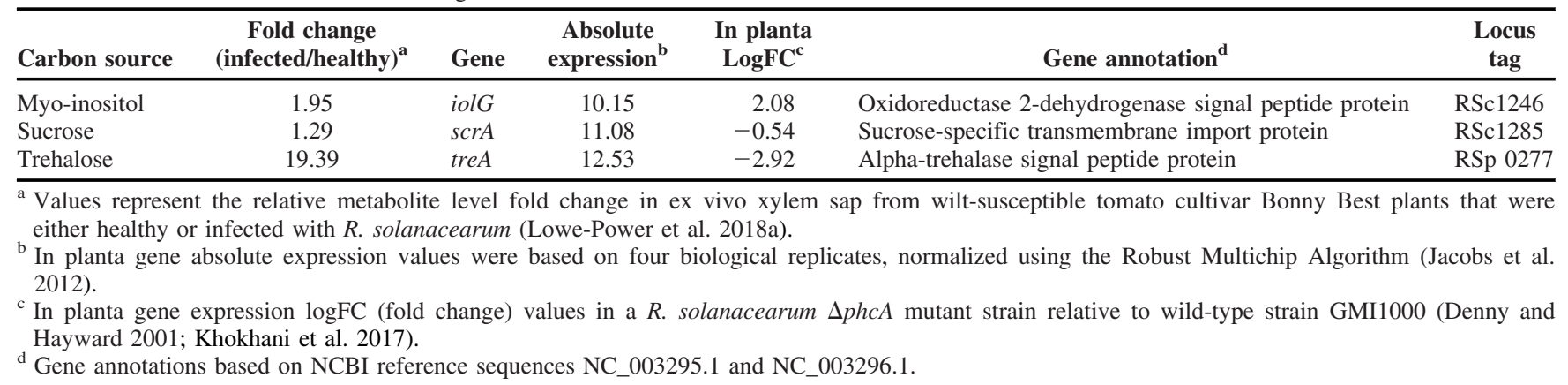


in growth on their respective targeted carbon source or sources. Complementing each mutation with a copy of the wild-type gene restored strain growth on the corresponding carbon source (Supplementary Table S1). These functional experiments confirmed the gene annotations of iolG, scrA, and treA and showed that the mutants could not use the corresponding sugars.

\section{Myo-inositol catabolism is required for colonization and competitive fitness early in tomato root infection.}

By $24 \mathrm{~h}$ after they reach a host root surface, $R$. solanacearum cells are multiplying in root cortical tissue and beginning to colonize root vascular bundles, but many have not yet reached the PhcA QS threshold of $1 \times 10^{7} \mathrm{CFU} / \mathrm{ml}$; this timepoint thus represents early disease (Caldwell et al. 2017; McGarvey et al. 1999). By $72 \mathrm{~h}$ postinoculation (hpi), many vascular elements are densely colonized and most $R$. solanacearum populations have transitioned into the HCD gene expression pattern, corresponding to the later stage of disease. Because $R$. solanacearum upregulates the myo-inositol pathway at LCDs, we hypothesized that consuming this sugar increases bacterial success at the earliest step in the infection process. We tested this by placing wild type, $\Delta p h c A, \Delta s c r A, \Delta$ treA, or $\Delta i o l G$ cells onto the root surface of tomato seedlings, allowing infection to proceed for 24 or $72 \mathrm{~h}$ and then quantifying $R$. solanacearum population sizes inside surface-sterilized roots in the root endosphere (Fig. 1). As expected, very early in root infection the LCD-locked $\Delta p h c A$ mutant was more successful than wild type but by $72 \mathrm{hpi}$, $\Delta p h c A$ populations in the root endosphere were smaller than those of the wild-type strain. Because it can use a wider range of nutrients and does not synthesize metabolically costly virulence factors, the $\Delta p h c A$ mutant grows faster in the earliest stages of plant infection (Khokhani et al. 2017; Peyraud et al. 2016). In contrast, subpopulations of wild-type cells experience intermittent HCDs and associated QS-mediated regulation that slows their growth as they fill xylem vessels and cortical tissue. However, the $\Delta p h c A$ growth advantage in roots is short-lived, likely because virulence factor production is critical for success in planta. The $\Delta s c r A, \Delta$ treA, and the $\Delta s c r A /$ treA double mutant reached population sizes similar to those of wild type, suggesting that $R$. solanacearum success during the first $24 \mathrm{~h}$ of root infection does not depend on access to sucrose or trehalose. However, at 24 hpi the iolG mutant was 92-fold reduced in root colonization compared with wild type, indicating that myo-inositol consumption is important early in the root colonization process. In contrast, mutants unable to use either or both sucrose and trehalose reached smaller population sizes in seedling roots 72 hpi (Fig. 1).

To further quantify the contribution of specific sugars to $R$. solanacearum success early in root infection, we coinoculated roots with wild type and mutant strains to determine if the catabolic mutants were reduced in competitive fitness. Tomato seedling roots were surface-inoculated, as described above, with a 1:1 mixture of wild-type and either $\Delta s c r A, \Delta$ treA, or $\Delta i o l G$ and the population sizes of each strain inside root endospheres were quantified at 24 and 72 hpi (Table 2). The $\Delta i o l G$ mutant was out-competed by the wild-type strain by 87 -fold in roots $24 \mathrm{hpi}$. At $72 \mathrm{hpi}$, there were no differences among strains in competitive fitness (Table 2). Together, these results indicate that the ability to use myo-inositol helps $R$. solanacearum multiply very early in plant infection while consuming sucrose and trehalose increases bacterial success only after the pathogen has reached high densities later in root colonization.

\section{Sucrose catabolism is required for colonization and} competitive fitness in tomato stems.

After $R$. solanacearum cells colonize root xylem vessels they spread rapidly up into the host stem. At this point in bacterial wilt disease development, most pathogen cells are in subpopulations that have surpassed the PhcA QS threshold of $1 \times 10^{7} \mathrm{CFU} / \mathrm{ml}$ (Genin and Denny 2012). Xylem sap metabolomics and in-planta transcriptomics suggest that trehalose and sucrose could be key carbon sources for $R$. solanacearum cells multiplying in tomato stems. To directly measure the contribution of specific xylem carbon sources to bacterial

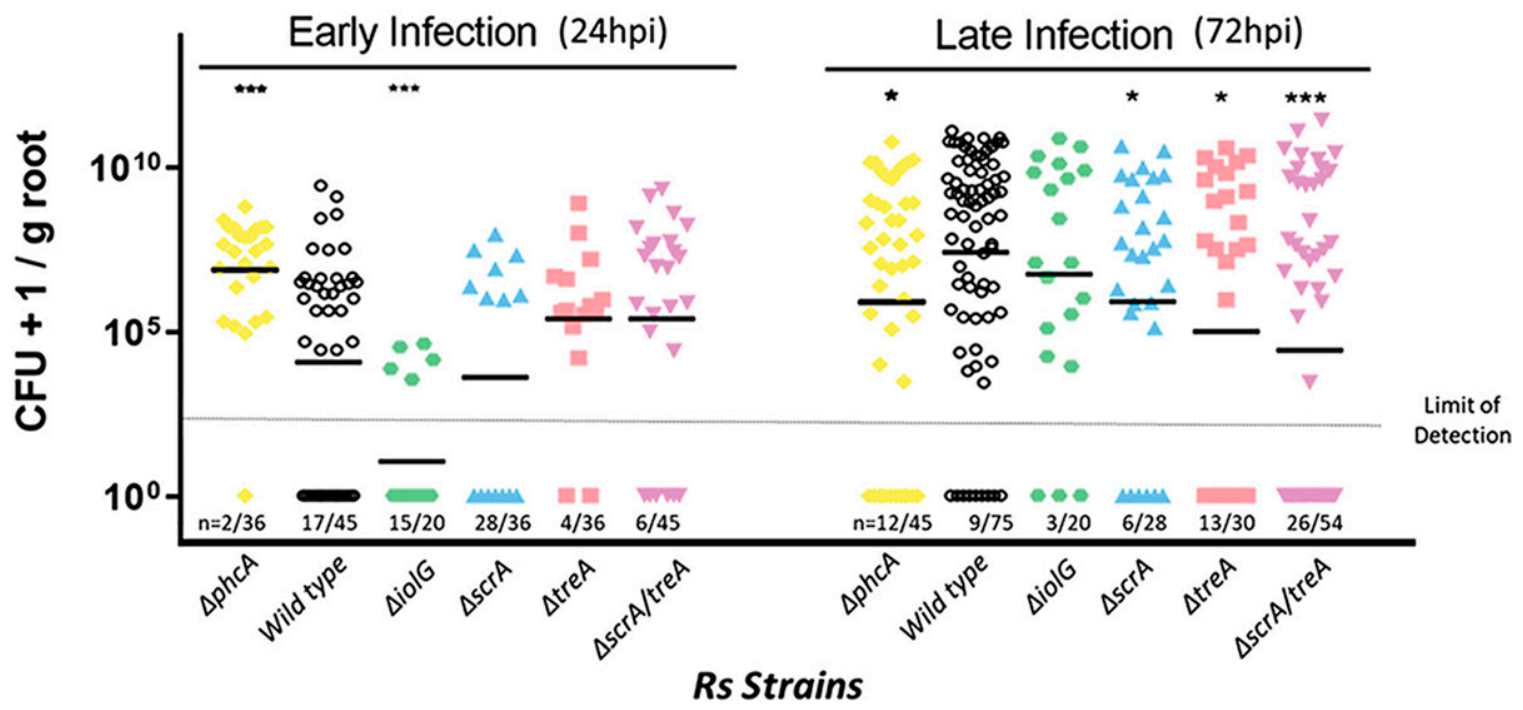

Fig. 1. A Ralstonia solanacearum (Rs) mutant unable to catabolize myo-inositol colonized the root endosphere poorly at 24 h postinoculation (hpi), while mutants unable to use sucrose or trehalose showed defects only at $72 \mathrm{hpi}$. The roots of 4-day-old unwounded tomato seedlings were inoculated with 2,000 cells of $R$. solanacearum $\Delta p h c A$ (yellow symbols), wild-type GMI1000 (black), $\Delta i o l G$ (green), $\Delta s c r A$ (blue), $\Delta$ treA (red), or $\Delta s c r A / t r e A$ (magenta). Internal bacterial population sizes were quantified by serial dilution plating of ground surface-sterilized roots harvested 24 or 72 hpi. Population size is shown as $\mathrm{CFU}+1$ to account for zero in the $\log _{10}$ scale. Each symbol represents the population size in one two-root sample; data shown represent three to six biological replicates. The number under each data column indicates the number of samples that did not contain any detectable bacteria out of the total sample number. Horizontal black lines represent the geometric mean population size of each strain. The gray dotted line indicates the assay limit of detection of $1 \times 10^{2} \mathrm{CFU}$ per gram. Asterisks indicate differences from wild-type population size at the same timepoint; one asterisk $(*)$ indicates $P<0.001$, two (**) $P<0.0001$, and three (***) $P<0.00001$, Mann-Whitney test. 
success at this disease stage, we compared the population sizes in tomato midstems of wild type, $\Delta s c r A, \Delta$ treA, and $\Delta i o l G R$. solanacearum strains 5 days after soil soak inoculation. The population sizes of the $\Delta i o l G$ and $\Delta$ treA catabolic mutants were indistinguishable from those of the wild-type strain. However, neither the $\Delta s c r A$ nor $\Delta s c r A / t r e A$ mutant colonized stems as well as the wild-type strain (Fig. 2). Interestingly, the population size of the $\Delta s c r A /$ tre $A$ double mutant was significantly lower than that of the $\Delta s c r A$ single mutant $(P<0.001$, Mann-Whitney test). Together, these results suggest that sucrose consumption contributes quantitatively to $R$. solanacearum multiplication in stems but that the pathogen does not need to consume either myo-inositol or trehalose to reach wild-type population sizes at this disease stage.

To more precisely explore the contributions of these sugars to bacterial success in tomato midstems, we forced the catabolic

Table 2. Competitive fitness of Ralstonia solanacearum catabolic mutants during tomato root endosphere colonization

\begin{tabular}{|c|c|c|c|c|}
\hline Time postinoculation & Competing strains & Strain population size ${ }^{a}$ & Competitive index ${ }^{b}$ & $P$ value $^{\mathrm{c}}$ \\
\hline \multirow[t]{8}{*}{$24 \mathrm{~h}$} & Wild type & $4.50 \times 10^{5}$ & 0.2956 & $0.0171^{*}$ \\
\hline & $\Delta i o l G$ & $5.21 \times 10^{2}$ & & \\
\hline & Wild type & $4.88 \times 10^{5}$ & 1.91784 & 0.1512 \\
\hline & $\Delta s c r A$ & $1.00 \times 10^{6}$ & & \\
\hline & Wild type & $1.11 \times 10^{7}$ & 1.93507 & 0.5252 \\
\hline & $\Delta$ treA & $1.61 \times 10^{8}$ & & \\
\hline & Wild type & $4.34 \times 10^{6}$ & 0.81981 & 0.5954 \\
\hline & $\Delta$ scrA/treA & $7.39 \times 10^{6}$ & & \\
\hline \multirow[t]{8}{*}{$72 \mathrm{~h}$} & Wild type & $1.62 \times 10^{6}$ & 1.482 & 0.2017 \\
\hline & $\Delta i o l G$ & $1.27 \times 10^{7}$ & & \\
\hline & Wild type & $2.75 \times 10^{8}$ & 0.86104 & 0.8319 \\
\hline & $\Delta s c r A$ & $3.08 \times 10^{8}$ & & \\
\hline & Wild type & $3.30 \times 10^{7}$ & 1.00095 & 0.6379 \\
\hline & $\Delta$ treA & $5.78 \times 10^{6}$ & & \\
\hline & Wild type & $6.98 \times 10^{8}$ & 0.79637 & 0.8296 \\
\hline & $\Delta s c r A /$ treA & $5.72 \times 10^{8}$ & & \\
\hline
\end{tabular}

${ }^{a}$ Bacterial population sizes determined by serial dilution plating of surface-sterilized ground seedling roots, shown as geometric mean of CFU per gram of root.

${ }^{b}$ Competitive index values represent the proportional competitive advantage of the competing strain over the wild-type GMI1000 reference strain. A competitive index of 1 indicates no competitive advantage between strains.

${ }^{\mathrm{c}} P$ values were determined by paired T-test. Population sizes are the geometric means of four biological replicate experiments, each containing 30 to 60 tomato seedling roots.

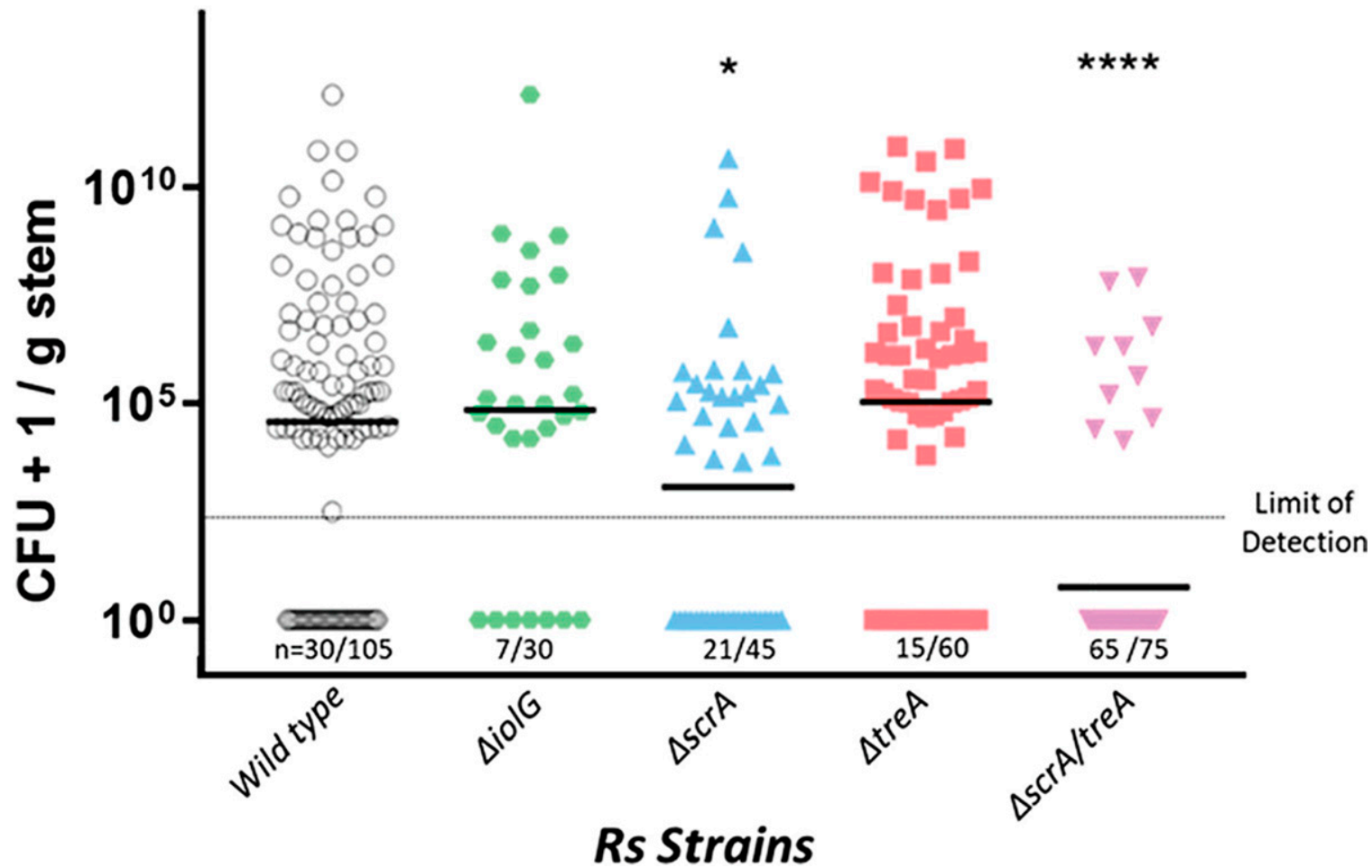

Fig. 2. Sucrose catabolism helps Ralstonia solanacearum (Rs) colonize tomato stems. Unwounded 17-day-old tomato plants were inoculated by soaking the soil with a $5 \times 10^{7} \mathrm{CFU} / \mathrm{ml}$ suspension of wild-type $R$. solanacearum (black), $\Delta i o l G$ (green), $\Delta s c r A$ (blue), $\Delta$ treA (red), or $\Delta s c r A / t r e A$ (magenta), After 5 days, the bacterial population size in each plant was determined by dilution plating a ground stem section harvested just above the cotyledon. Population size is displayed as CFU +1 to account for zeroes in the $\log _{10}$ scale. Each symbol represents the population size in one plant. Data represent three to six independent experiments. The number under each data column indicates the number of samples that did not contain any detectable bacteria to the total sample number. Horizontal black lines represent the geometric mean of each strain's population size. The gray dotted line indicates the assay limit of detection of $1 \times 10^{2} \mathrm{CFU}$ per gram. Asterisks indicate population sizes different from the wild type (one asterisk [*] $P<0.05$, four [****] $P<0.0001$, Mann-Whitney test). 
mutants to directly compete with the wild-type strain by coinoculating tomato plants through a cut leaf petiole with a 1:1 mixture of wild-type GMI1000 and either $\Delta s c r A, \Delta$ treA, or $\Delta i o l G$. This method bypasses roots by introducing the bacteria directly into stem xylem vessels. At 5 days after inoculation, the $\Delta s c r A$, $\Delta$ treA, and $\Delta s c r A /$ treA catabolic mutants were out-competed by wild-type GMI1000 (Table 3). In contrast, loss of myo-inositol catabolizing ability did not affect competitive fitness under these conditions. This indicates that the ability to catabolize xylem sucrose provides $R$. solanacearum with a significant fitness advantage during stem infection and, also, that ability to degrade trehalose increases pathogen competitive fitness at midstage disease.

\section{Sucrose and trehalose catabolism contribute additively} to bacterial wilt virulence.

To assess the contributions of myo-inositol, sucrose, and trehalose across the entire bacterial wilt disease process, we measured virulence of the four catabolic mutants following a naturalistic soil-soak inoculation of tomato plants. Consistent with the finding that, by 3 days postinoculation (dpi), the $\Delta i o l G$ mutant colonized roots as well as wild type, in this holistic assay, $\Delta i o l G$ was as virulent as its wild-type parent (Fig. 3). However, the $\Delta s c r A$ and $\Delta$ treA single mutants were each defective in virulence compared with wild type. Further, deleting both $s c r A$ and tre $A$ reduced virulence more than mutating either gene alone. This suggests that catabolism of sucrose and of trehalose contribute additively to $R$. solanacearum virulence.
Ex vivo xylem sap from $\Delta s c r A$-infected plants supports more bacterial growth.

When catabolic $R$. solanacearum mutants colonize plants, they would be expected to leave unconsumed nutrients in xylem sap. We tested this hypothesis by culturing bacteria in $50 \mu \mathrm{l}$ of

Table 3. Competitive fitness of Ralstonia solanacearum catabolic mutants during colonization of tomato stems following cut petiole inoculation

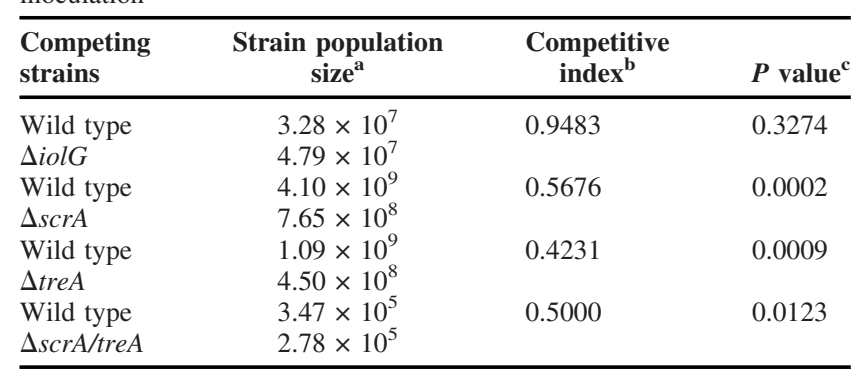

${ }^{a}$ Bacterial population sizes determined by serial dilution plating of ground stems, shown as geometric mean of CFU per gram of stem.

b Competitive index values represent the proportional competitive advantage of the competing strain over the wild-type GMI1000 reference strain. A competitive index of 1 indicates no competitive advantage between strains.

${ }^{\mathrm{c}} P$ values were determined by paired T-test. Population sizes are the geometric means of three $(\Delta \operatorname{iol} G)$ or four $(\Delta s c r A, \Delta$ treA, and $\Delta s c r A$ treA) biological replicate experiments, each containing midstem sections from 30 to 60 tomato plants.

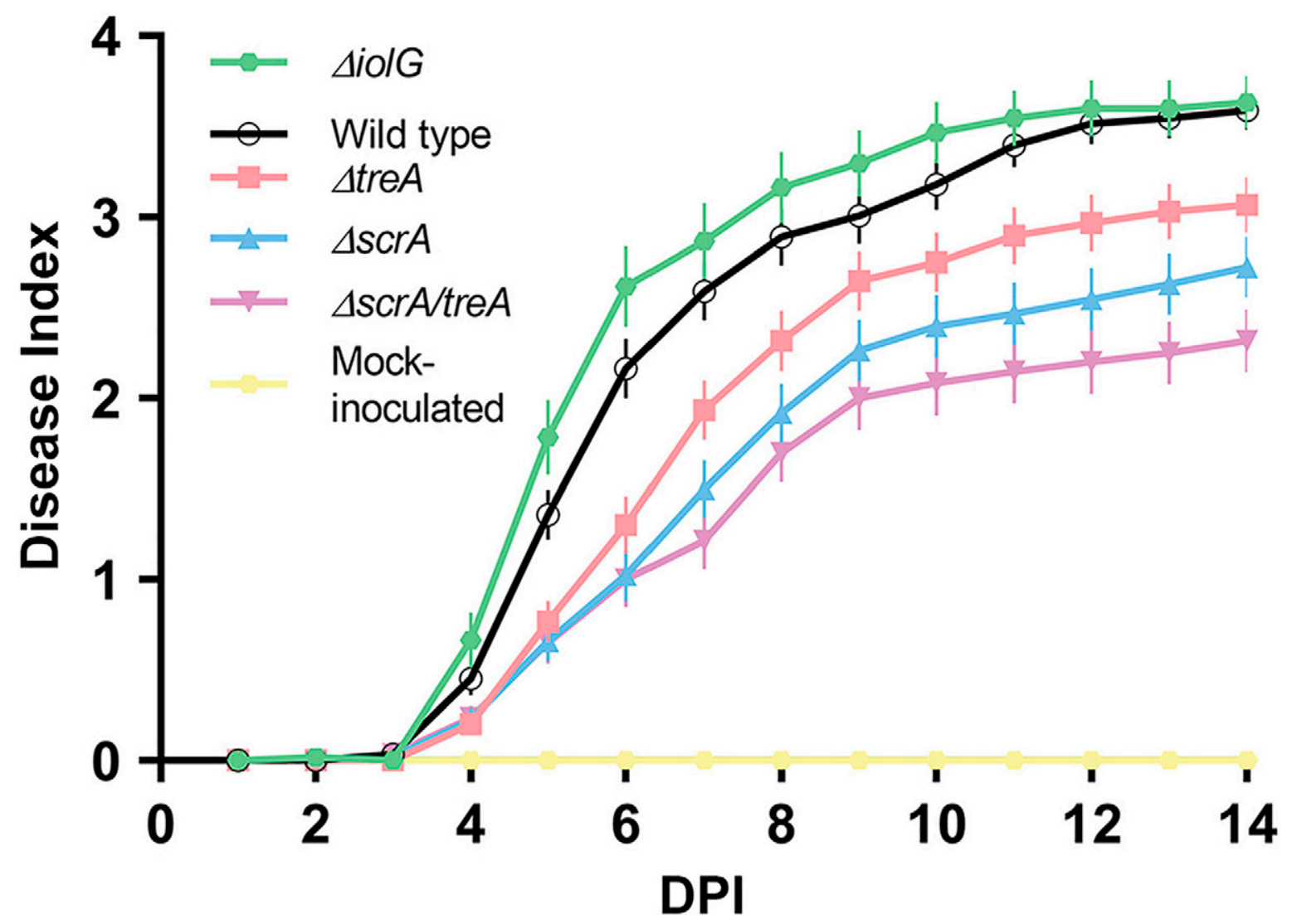

Fig. 3. Ralstonia solanacearum needs catabolism of sucrose and trehalose for full bacterial wilt virulence. Unwounded 17-day-old tomato plants were inoculated by pouring a dilute bacterial suspension into the pot to a final pathogen concentration of $5 \times 10^{7} \mathrm{CFU}$ per gram of potting mix. Plants were incubated in a $28^{\circ} \mathrm{C}$ growth chamber and symptoms were rated daily on a 0 to 4 disease index. Data shown are the means of four independent experiments, each containing 15 plants per treatment, except the wild-type data, which reflect eight independent experiments. The $\Delta$ treA, $\Delta s c r A$, and $\Delta s c r A /$ treA mutants were less virulent than wild-type $R$. solanacearum $(P<0.002$, repeated measures analysis of variance [ANOVA]). Virulence of the $\Delta i o l G$ catabolic mutant was not different from that of wild-type strain GMI1000 $(P=0.306$, repeated measures ANOVA). Error bars show standard error of the mean disease index reading for each day. 
filter-sterilized xylem sap harvested from plants that had been infected 5 days earlier with either wild type or mutant $R$. solanacearum strains. The ex vivo xylem sap was prepared by pooling harvested sap normalized to host plant colonization levels of $5 \times 10^{6} \mathrm{CFU}$ per gram. Wild type, $\Delta i o l G, \Delta s c r A, \Delta t r e A$, and $\Delta s c r A /$ treA strains all grew equally well on sap from plants previously infected with wild type, $\Delta i o l G$, or $\Delta$ treA bacteria (Fig. 4). However, all $R$. solanacearum strains grew better on sap from plants infected with $\Delta s c r A$ or $\Delta s c r A / t r e A$ mutants, which cannot catabolize sucrose. This indicates that the increased bacterial growth was fueled by sucrose, which was present at around $270 \mu \mathrm{M}$ in sap from wild type-infected plants (Fig. 5). Interestingly, sap from plants infected with the $\Delta s c r A$ / treA double mutant supported more $R$. solanacearum growth than sap from plants infected with $\Delta s c r A$ or $\Delta$ treA single mutants. This increased growth might have been fueled by catabolism of trehalose. However, direct measurement of trehalose showed that xylem sap from wild type-infected plants contained less than $100 \mathrm{nM}$ trehalose, which was the detection limit of the assay. $R$. solanacearum GMI1000 can grow on trehalose as a sole carbon source only at concentrations above $1 \mathrm{mM}$ (Supplementary Table S2). Thus, the concentration of trehalose in tomato xylem sap was too low to support bacterial growth, so catabolism of trehalose as a carbon source cannot explain the additive growth defect in the $\Delta s c r A / t r e A$ double mutant.

To directly test the hypothesis that sucrose-nonmetabolizing $R$. solanacearum mutants leave this nutrient unconsumed when they infect plants, we measured sucrose concentrations in ex vivo xylem sap harvested from plants infected by various strains. Sap from plants infected with wild type or $\Delta i o l G$ contained similar levels of sucrose, around $270 \mu \mathrm{M}$, while sap from plants infected with $\Delta s c r A$ or $\Delta s c r A /$ treA contained $43 \%$ more sucrose than sap from wild type-infected plants (Fig. 5). Unexpectedly, sap from plants infected with the $\Delta$ treA single mutant also contained higher sucrose levels than sap from wild-type plants. Although sap from plants previously infected by $\Delta s c r A$ / treA supported the best growth of wild-type $R$. solanacearum (Fig. 4), bacterial growth in sap from plants previously infected by $\Delta s c r A$ was the most different from growth in sap from plants infected with wild-type $R$. solanacearum. This suggests that, while an increase in sucrose is responsible for some increased growth in ex vivo sap, the additive growth effect of the $\Delta t r e A$ and $\Delta s c r A$ deletions is not fully explained by increase in sucrose concentration. Together, these two experiments suggest that sucrose is a key nutrient supporting $R$. solanacearum growth in xylem, while trehalose improves the xylem sap environment for $R$. solanacearum via a nonnutritive mechanism.

\section{DISCUSSION}

Whole-genome expression studies indicated that $R$. solanacearum uses density-dependent QS to mediate a tradeoff from a broad metabolic capacity at LCD to a much narrower catabolic menu at HCD (Khokhani et al. 2017; Peyraud et al. 2016). This shift parallels the transition between early- and late-stage plant infections. Comparing the growth of $R$. solanacearum and a QS mutant on many substrates revealed the counterintuitive fact that many nutrients are not accessible to $R$. solanacearum cells once

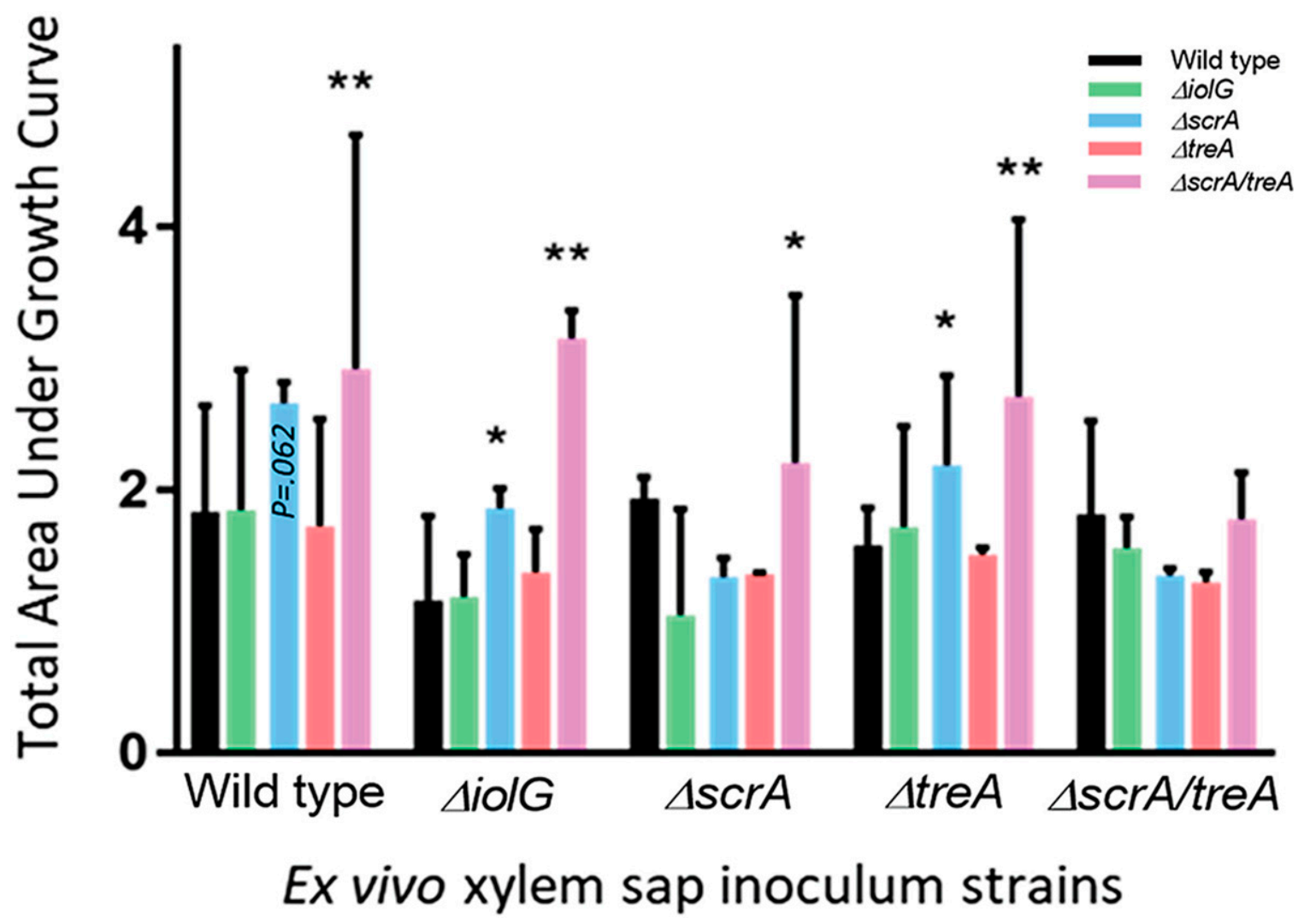

Fig. 4. Ralstonia solanacearum strains able to use sucrose grew better on ex vivo xylem sap from plants infected with sucrose nonmetabolizing mutants. Growth over $36 \mathrm{~h}$ of various $R$. solanacearum strains in filter-sterilized xylem sap, from plants previously infected by either wild-type $R$. solanacearum (black), $\Delta$ iolG (green), $\Delta s c r A$ (blue), $\Delta$ treA (red), or $\Delta s c r A / t r e A$ (magenta) strains, was measured using a plate reader and is shown as area under the growth curve. This experiment was replicated three to six times, with each experiment containing three technical replicates per treatment. Error bars indicate the standard error of the mean growth value for all nine to 18 independent growth curves. Asterisks indicate difference from growth of the wildtype strain on the same xylem sap source; one asterisk (*) indicates $P<0.05$, two $\left(^{* *}\right) P<0.01$, Mann-Whitney test. A trending $P$ value of $P=0.062$ is shown for wild-type cells growing in sap from $\Delta s c r A$-inoculated plants. 
they reach $\mathrm{HCD}$, including nutrients like myo-inositol that are known to be available in planta. If a regulatory circuit forces the bacterium to forgo available food sources, $R$. solanacearum must benefit from restricting its metabolic capacity to only a subset of nutrients, possibly by avoiding the cost of synthesizing many catabolic proteins. We experimentally tested this model and found that an $\Delta i o l G$ mutant, which cannot use myo-inositol, is reduced in root endosphere colonization but recovers once bacteria reach HCD and $\Delta i o l G$ ultimately can cause disease as well as wild type. This shows that myo-inositol is a key nutrient early in infection when $R$. solanacearum has access to its full metabolic capacity, but once the bacteria reach HCD, they do not need myo-inositol to succeed even though it is present in xylem sap.

Myo-inositol catabolism is not the only pathway that $R$. solanacearum represses at HCD. Transcriptome profiles of $R$. solanacearum $\Delta p h c A$ mutants, which are genetically locked in LCD mode, indicated that wild-type $R$. solanacearum had lower expression of 255 genes, including many catabolic and transporter genes (Khokhani et al. 2017; Perrier et al. 2018). These genes encode uptake and breakdown of core metabolites like sugars, amino acids, and fatty acids but, also, secondary compounds like hydroxycinnamic acid and galacturonic acid. Some of these metabolites are available to $R$. solanacearum in the xylem. As we observed for myo-inositol, the ability to degrade hydroxycinnamic acid contributes to early root colonization by $R$. solanacearum but not to bacterial fitness in the stem (Lowe et al. 2015). However, this contribution may be explained by counter-defense rather than nutrition. Although some $R$. solanacearum strains can use hydroxycinnamic acids as a sole carbon source, they are also toxic to $R$. solanacearum at high concentrations (Lowe-Power et al. 2016). Another potential nutrient source is provided by degradation of cell-wall components like pectin, a polymeric matrix of primarily galacturonic acid (Abbott and Boraston 2008). R. solanacearum degrades this complex and abundant polymer with an array of extracellular enzymes, including a pectin methylesterase and three polygalacturonases (Tans-Kersten et al. 1998). Deleting these enzymes either individually or together only slightly decreased virulence on tomato by either $R$. solanacearum GMI1000 or the phylotype I strain K60 (Gonzales and Allen 2003; Huang and Allen 2000; Liu et al. 2005; Tans-Kersten et al. 1998). However, these studies did not measure effects of pectinase mutations on root colonization. While galacturonic acid is present in xylem sap from $R$. solanacearum-infected tomato, expression of the secreted pectinases and the catabolic pathway that converts galacturonic acid to pyruvate is repressed at HCD in planta and in culture (Khokhani et al. 2017; Lowe-Power et al. 2018a; Mori et al. 2017; Perrier et al. 2018). Infection stage-specific experiments could determine if degrading metabolites like hydroxycinnamic acid and galacturonic acid contribute to $R$. solanacearum virulence early in disease.

$R$. solanacearum cells living in rhizospheres are likely relatively few and not at quorum density. When these LCD-mode bacteria encounter plant root exudates, they can catabolize many compounds in this rich cocktail. Exudates contain metabolites available to LCD-mode $R$. solanacearum, including sugars like myo-inositol, amino acids, and larger molecules and polymers like fatty acids and glycerophosphoinositide membrane lipids that require myo-inositol as a building block (Bais et al. 2006; Čepulytė et al. 2018; Korenblum et al. 2020; Vílchez et al. 2020; Zhalnina et al. 2018). Plant nucleic acids offer another

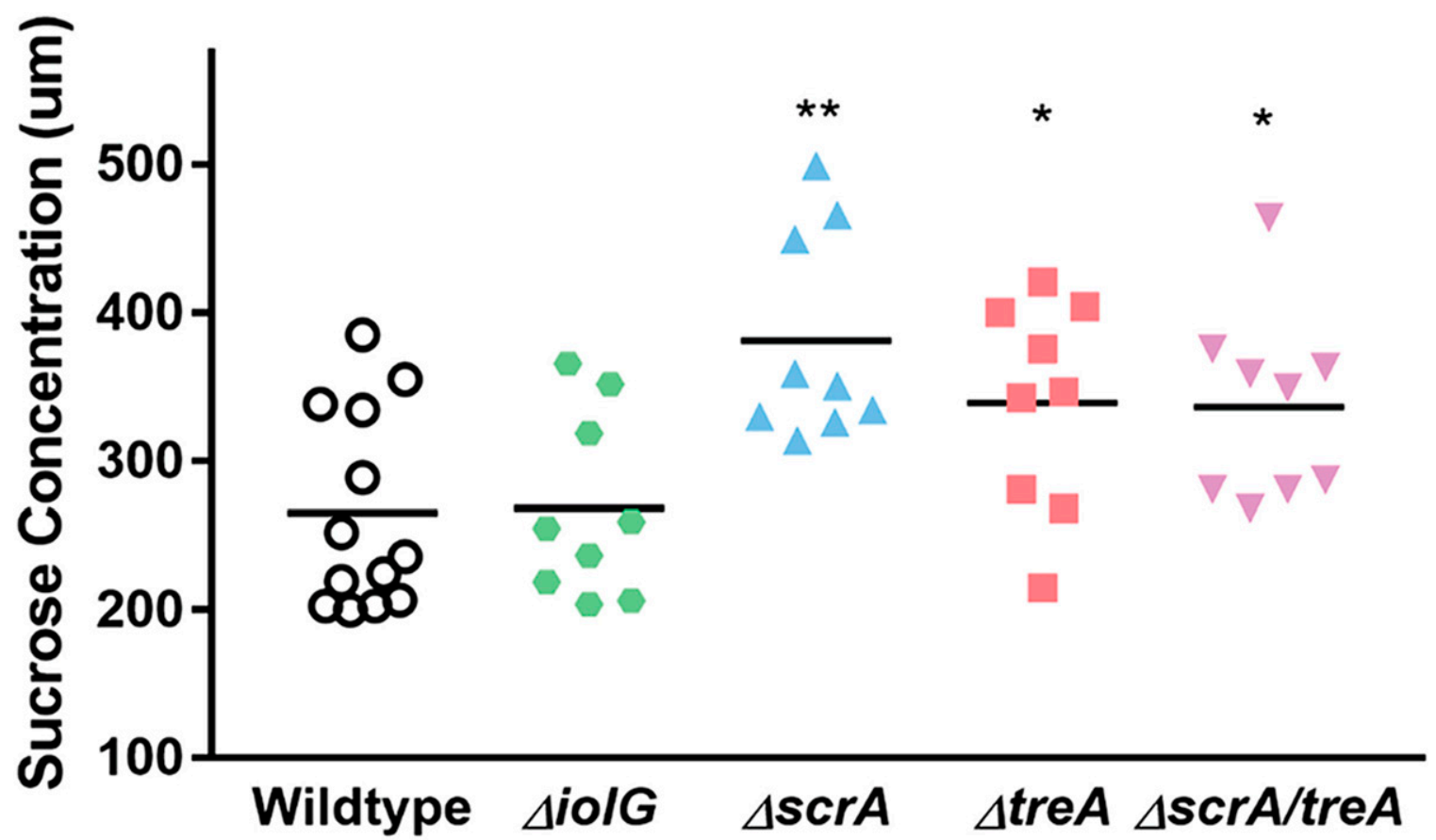

\section{Strains}

Fig. 5. Infection with Ralstonia solanacearum catabolic mutants $\Delta s c r A, \Delta t r e A$, and $\Delta s c r A / t r e A$ modulates sucrose levels in tomato xylem. Sucrose was quantified in ex vivo xylem sap using the GOD/invertase method (Teixeira et al. 2012). Each plate included 10 to $1,000 \mu \mathrm{M}$ sucrose standards that were used to construct a calibration curve to determine the sucrose concentration in each sample. Each point represents the mean of triplicate technical replicates; analysis was done on at least three biological replicate experiments. One asterisk $\left(^{*}\right)$ indicates $P<0.01$, two $(* *) P<0.001$, Students paired Ttest. There was no significant difference between mutant strains $\Delta$ treA or $\Delta s c r A / t r e A$ when compared with $\Delta s c r A(P=0.23$ and $P=0.18$, respectively, Students paired T-test). 
potential nutrient source in the rhizosphere. DNA secreted from root border cells plays an important role in blocking entry of root pathogens, but $R$. solanacearum secretes DNAses to cut through these underground nets (Driouich et al. 2013; Tran et al. 2016). These extracellular DNAses facilitate $R$. solanacearum entry and spread within hosts, and they may also release a nutrient source in the rhizosphere. Consistent with this, an LCDlocked $\Delta p h c A$ mutant grows better than wild type on guanine and adenine (Khokhani et al. 2017; Tran et al. 2016). We speculate that, like myo-inositol, many carbon and nitrogen sources that $R$. solanacearum cannot metabolize at HCD contribute to $R$. solanacearum success early in disease.

After $R$. solanacearum multiplies in the confined spaces inside plants and reaches quorum cell densities, the bacterium can use a much narrower set of metabolites, notably sucrose (Khokhani et al. 2017). Sucrose is the most common sugar in plant roots and shoots (Aubry et al. 2019). We found that $R$. solanacearum did not need the ability to catabolize sucrose for successful root colonization, but it was critically important later in pathogenesis, as is also true for phylotype II strain $R$. solanacearum UW551 (Jacobs et al. 2012). Relative to wild type, an $\Delta s c r A$ mutant was reduced in colonization and competitive fitness in midstems and was less virulent. Even though xylem vessels of infected plants contain very large populations of bacteria avidly consuming sucrose, xylem sucrose levels remained surprisingly high during infection. Sucrose levels were even higher if the infecting strain could not use sucrose. These results suggest that $R$. solanacearum infection somehow causes plants to direct sucrose into xylem vessels, possibly by means of type 3-secreted effectors. It is not uncommon for plant pathogens to manipulate their hosts to acquire nutrients. Xanthomonas spp. use TAL effectors to upregulate host sucrose-transporting SWEET genes (Chen et al. 2010; Cohn et al. 2014; Cox et al. 2017). R. solanacearum effectors have been shown to directly influence host metabolism of nutrients like GABA and putrescine (Wu et al. 2019; Xian et al. 2019 ) and a $R$. solanacearum effector may direct host production of trehalose-6-phosphate (Poueymiro et al. 2014).

Alternatively, plants may direct sucrose to the xylem in an attempt to combat water stress and xylem blockage associated with bacterial wilt disease. This phloem-loading hypothesis suggests that plants can repair embolisms (breaks in the xylem vessel water column) by moving solutes like sugars from the phloem into the xylem. These solutes, combined with the action of plant aquaporins, can drive water osmotically into the xylem, refilling the vessel and also enriching it in nutrients (Knipfer et al. 2016; Nardini et al. 2011). While some xylem-dwelling bacterial pathogens like Xylella fastidiosa are known to induce embolism formation in their host, it is not known if $R$. solanacearum also has this ability (Lowe-Power et al. 2018b; Pérez-Donoso et al. 2007). Because of its clear importance to $R$. solanacearum success in the xylem, it will be important to determine the mechanism by which sucrose moves into xylem vessels during bacterial wilt disease.

Trehalose is emerging as an important factor in bacterial wilt disease. Its catabolism by the TreA trehalase is strongly upregulated at $\mathrm{HCD}$ both in culture and in planta (Khokhani et al. 2017; Mori et al. 2017; Perrier et al. 2018). Trehalose levels in tomato xylem increased 19-fold upon $R$. solanacearum infection, and we previously showed that the trehalose in xylem sap is produced by the plant, not by the bacterium (Lowe-Power et al. 2018a; MacIntyre et al. 2020). It is unlikely that trehalose contributes to $R$. solanacearum virulence by acting as a carbon source. Using enzymatic assays with limits of detection below the $1 \mathrm{mM}$ threshold required to support $R$. solanacearum growth, we were unable to detect trehalose in xylem sap from plants infected with $R$. solanacearum wild type, $\Delta s c r A, \Delta t r e A$, or $\Delta s c r A / t r e A$ strains (data not shown). Nonetheless, an $\Delta s c r A$ /
treA double mutant did not colonize tomato plants as well as wild type or even as well as single mutants lacking either scrA or treA. Further, the double mutant had a greater virulence defect than either single mutant. In coinoculation experiments, $R$. solanacearum wild type and the $\Delta s c r A / \Delta t r e A$ double mutant strain both consistently reached a lower mean population size when they were competing with each other in planta following cut-petiole inoculation (Table 3 ). No such population size difference was evident in the biologically different root endosphere competitive fitness experiment (Table 2). This result, which was consistent over four biological replicate experiments, may offer a clue about why $\Delta$ treA mutants are less successful in tomato stems even though trehalose is not a significant carbon source for $R$. solanacearum in planta. Finally, xylem sap collected from $\Delta s c r A / t r e A$-infected tomato plants supported more $R$. solanacearum growth than sap from $\Delta s c r A$-infected plants even though it contained less sucrose. This suggests that trehalose degradation increases xylem sap nutrient value independently of sucrose. We suspect that the small quantities of trehalose in tomato xylem sap affect plant responses in ways that increase $R$. solanacearum growth. Trehalose is known to modulate plant metabolic regulation and stress tolerance (Lunn et al. 2014; Paul et al. 2008). Its degradation may contribute to $R$. solanacearum virulence by combating osmotic stress or manipulating host physiology. Arabidopsis plants use trehalose 6-phoshate as a signal to balance sucrose to starch storage ratios in leaves (Kolbe et al. 2005). $R$. solanacearum may use TreA to degrade this plant signaling molecule and thus maintain an optimal nutrient enrichment of tomato xylem sap. Alternatively, in a separate study, we found evidence that trehalose can act as a DAMP (damage-inducible molecular pattern) that increases tomato defenses. $R$. solanacearum may thus degrade trehalose in xylem sap to avoid sparking plant defenses (A. M. MacIntyre and C. Allen unpublished).

When growing at LCDs in the uniform environment of a shaking culture flask, the $\Delta p h c A$ mutant and wild-type cells are in the same LCD regulatory state and initially grow at the same rate. So why does the $\Delta p h c A$ mutant multiply faster than wildtype $R$. solanacearum early in the plant infection process? This is likely explained by the heterogenous microenvironments that $R$. solanacearum inhabits inside roots. During root colonization, $R$. solanacearum cells are not at uniform population density and thus are not in a uniform regulatory state. Surprisingly small populations of bacteria can attain quorum in confined conditions in which QS signal cannot diffuse away. An in-situ gene expression analysis showed that aggregates as small as 33 cells reach quorum when the epiphytic bacterium Pseudomonas syringae is enclosed in an EPS matrix (Dulla and Lindow 2008).

Only a few hours after inoculation, $R$. solanacearum cells have already filled some vessels or intercellular spaces in the tomato root cortex (Vasse 1995). As they multiply and spread in host tissues, $R$. solanacearum subpopulations reach quorum intermittently when QS signal accumulates in these small spaces. The resulting QS-mediated downregulation of catabolic pathways slows their growth while QS-mediated upregulation of virulence functions enables long-term success inside the host. In contrast, the $\Delta p h c A$ mutant does not respond to QS signal and so it grows uniformly until it is limited by an inability to produce virulence factors.

Taken together, our results suggest that $R$. solanacearum central metabolism is highly dynamic. The catabolic profile of the pathogen changes over successive infection stages to optimize growth and virulence. As $R$. solanacearum cells experience habitats with diverse and scarce nutrients, they cast a wide net by expressing many different catabolic pathways. We show that $R$. solanacearum uses myo-inositol, one of many nutrients present in tomato root exudate, to multiply in the rhizosphere and early in plant infection. As wilt disease progresses, xylem-dwelling $R$. 
solanacearum cells focus their metabolism to use sucrose as a key energy source while degrading trehalose to manipulate plant responses in ways that favor the pathogen. Studies are underway to better understand the specific roles of trehalose in plant drought stress responses and bacterial wilt disease resistance.

\section{MATERIALS AND METHODS}

\section{Bacterial strains and culture.}

Bacterial strains used in this research are listed in Supplementary Table S3. $R$. solanacearum strains were routinely cultured as previously described (Khokhani et al. 2018). Bacterial growth was measured spectrophotometrically as optical density at 600 $\mathrm{nm}\left(\mathrm{OD}_{600}\right)$, using a Bio-Tek microplate reader.

Ex vivo xylem sap to be used as a bacterial growth medium or for sugar analysis was harvested from bacterial wilt-susceptible cultivar Bonny Best tomato plants 5 dpi as described, with modifications (Khokhani et al. 2018; Lowe-Power et al. 2018a). $R$. solanacearum population sizes were measured in each sampled plant. Only sap from plants containing $5 \times 10^{4}$ to $5 \times 10^{8}$ CFU per gram of stem was sterilized by passage through a $0.22-$ micron filter and was retained for growth experiments. Samples were further normalized by pooling ex vivo sap samples to ensure they represented plants carrying an average bacterial population size of $5 \times 10^{6} \mathrm{CFU}$ per gram. To measure $R$. solanacearum growth in the ex vivo xylem sap used as a culture medium, a 1- $\mu$ l bacterial suspension, adjusted to an $\mathrm{OD}_{600}$ of 0.01 , was added to $50 \mu \mathrm{l}$ of pooled filter-sterilized sap in 96well flat-bottom half-area plates and was cultured at $28^{\circ} \mathrm{C}$ in a spectrophotometric microplate reader, which is a microaerophilic environment. Bacterial growth curves over $24 \mathrm{~h}$ were generated using a Bio-Tek microplate reader, measuring spectrophotometrically as $\mathrm{OD}_{600}$. Area under growth curve was analyzed, relative to growth of the wild-type strain, using the MannWhitney test.

\section{$R$. solanacearum deletion mutants.}

Cloning, restriction digestion, sequencing, and PCR were performed using standard methods (Castañeda et al. 2005; Heckman and Pease 2007; Monteiro et al. 2012). DNA sequencing was performed at the University of Wisconsin-Madison Biotechnology Center and oligonucleotide primers were synthesized by Integrated DNA Technologies. Gibson assembly was used to create in-frame deletion constructs with the primers listed in Supplementary Table S3. Deletion constructs were introduced into the chromosome of wild-type $R$. solanacearum GMI1000 by double homologous recombination, with $s a c B$ counterselection as described (Castañeda et al. 2005), to create

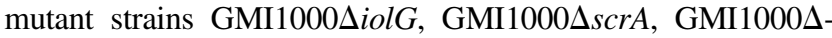
treA, and GMI1000 $\Delta$ scrA/treA. Complementation constructs were introduced into the selectively neutral att site in the chromosome of the relevant $R$. solanacearum mutant by double homologous recombination, as previously described,

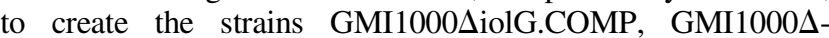
scrA.COMP, GMI1000 $\Delta$ treA.COMP, and GMI1000 $\Delta \mathrm{scrA} /$ treA.COMP (Monteiro et al. 2012). Correct allelic replacement for each mutant and complemented strain was confirmed by PCR and DNA sequencing.

\section{Plant assays.}

Virulence assays. Plants were inoculated using a naturalistic soil-soak method (Khokhani et al. 2018). Briefly, a $50 \mathrm{ml}$ of a diluted water suspension of bacteria was poured onto the soil of unwounded 17-day-old Bonny Best tomato plants growing in 4-inch pots at $28^{\circ} \mathrm{C}$ to achieve a final pathogen density of $5 \times$ $10^{7} \mathrm{CFU}$ per gram of soil. Data were analyzed using repeated measures analysis of variance (ANOVA), which is used for independent variables that are related because they are sampled repeatedly over time.

Plant colonization and competitive fitness assays. Bacterial colonization and quantification in plant stems was assessed as described (Khokhani et al. 2018). To measure the ability of $R$. solanacearum strains to colonize root endospheres, 4-day-old axenic tomato seedlings were inoculated by applying a $10-\mu \mathrm{l}$ water suspension containing 2,000 cells to seedling roots as described (Khokhani et al. 2018). Plants were inoculated with antibiotic resistance-marked wild type and marked mutant cell suspensions combined in a 1:1 ratio, using the root inoculation method described above. To remove bacteria from root surfaces, before tomato seedling roots were ground and plated, they were surface-sterilized by treatment with $10 \%$ bleach for $30 \mathrm{~s}, 70 \%$ ethanol for $30 \mathrm{~s}$, and were rinsed in sterile water for $1 \mathrm{~min}$. All experiments were repeated at least three times. A competitive index of $R$. solanacearum strains was calculated as previously described (Macho et al. 2007). Data were analyzed using a Mann-Whitney $U$ test or Wilcoxon sign-rank test.

Sucrose levels in xylem sap were measured using a modified GOD/invertase enzymatic assay (Teixeira et al. 2012). A 5- $\mu \mathrm{l}$ sap sample was added to $85 \mu \mathrm{l}$ of distilled water plus $10 \mu \mathrm{l}$ of invertase solution $(10 \mathrm{mg} / \mathrm{ml}$ [Sigma-Aldrich] in water, 300 units/mg) and a sample was placed in each well of a 96-well plate. The plate was sealed and incubated in a $55^{\circ} \mathrm{C}$ water bath for $10 \mathrm{~min}$. The plate was then removed from the water bath, $200 \mu \mathrm{l}$ of GOD reagent (Pointer Scientific Inc.) was added, the plate was sealed again, was incubated in a $37^{\circ} \mathrm{C}$ water bath for $15 \mathrm{~min}$, and was then placed at room temperature for $5 \mathrm{~min}$. The absorbance at $490 \mathrm{~nm}$ was read in a spectrophotometric plate reader. Each plate included 10 to $1,000 \mu \mathrm{mol}$ sucrose solution standards to make a calibration curve that was used to determine sucrose concentration in each sample. Each analysis was done in triplicate.

\section{AUTHOR-RECOMMENDED INTERNET RESOURCES}

NCBI reference sequences:

www.ncbi.nlm.nih.gov/nuccore/NC_003295.1 and

www.ncbi.nlm.nih.gov/nuccore/NC_003296.1

\section{ACKNOWLEDGMENTS}

The authors thank D. Khokhani and T. Lowe-Power for helpful discussions.

\section{LITERATURE CITED}

Abbott, D. W., and Boraston, A. B. 2008. Structural biology of pectin degradation by Enterobacteriaceae. Microbiol. Mol. Biol. Rev. 72:301-316.

Allen, C., Prior, P., and Hayward, A. C. 2005. Bacterial Wilt Disease and the Ralstonia solanacearum Species Complex. APS Press, St. Paul, MN, U.S.A..

Álvarez, B., Biosca, E. G., and López, M. M. 2010. On the life of Ralstonia solanacearum, a destructive bacterial plant pathogen. Pages $267-$ 279 in: Current Research, Technology and Education Topics In Applied Microbiology and Microbial Biotechnology. A. Mendez-Vilas, ed. Vol. 1. Formatex, Badajoz, Spain.

Aubry, E., Dinant, S., Vilaine, F., Bellini, C., and Le Hir, R. 2019. Lateral transport of organic and inorganic solutes. Plants 8:20.

Bais, H. P., Weir, T. L., Perry, L. G., Gilroy, S., and Vivanco, J. M. 2006. The role of root exudates in rhizosphere interactions with plants and other organisms. Annu. Rev. Plant Biol. 57:233-266.

Caldwell, D., Kim, B. S., and Iyer-Pascuzzi, A. S. 2017. Ralstonia solanacearum differentially colonizes roots of resistant and susceptible tomato plants. Phytopathology 107:528-536.

Castañeda, A., Reddy, J. D., El-Yacoubi, B., and Gabriel, D. W. 2005. Mutagenesis of all eight $a v r$ genes in Xanthomonas campestris pv. campestris had no detected effect on pathogenicity, but one avr gene affected race specificity. Mol. Plant-Microbe Interact. 18:1306-1317. 
Čepulyte, R., Danquah, W. B., Bruening, G., and Williamson, V. M. 2018. Potent attractant for root-knot nematodes in exudates from seedling root tips of two host species. Sci. Rep. 8:10847.

Chen, L. Q., Hou, B. H., Lalonde, S., Takanaga, H., Hartung, M. L., Qu, X. Q., Guo, W. J., Kim, J. G., Underwood, W., Chaudhuri, B., Chermak, D., Antony, G., White, F. F., Somerville, S. C., Mudgett, M. B., and Frommer, W. B. 2010. Sugar transporters for intercellular exchange and nutrition of pathogens. Nature 468:527-532.

Cohn, M., Bart, R. S., Shybut, M., Dahlbeck, D., Gomez, M., Morbitzer, R., Hou, B. H., Frommer, W. B., Lahaye, T., and Staskawicz, B. J. 2014. Xanthomonas axonopodis virulence is promoted by a transcription activator-like effector-mediated induction of a SWEET sugar transporter in cassava. Mol. Plant-Microbe Interact. 27:1186-1198.

Coplin, D. L., Sequeira, L., and Hanson, R. S. 1974. Pseudomonas solanacearum: Virulence of biochemical mutants. Can. J. Microbiol. 20:519-529.

Cox, K. L., Meng, F., Wilkins, K. E., Li, F., Wang, P., Booher, N. J., Carpenter, S. C. D., Chen, L. Q., Zheng, H., Gao, X., Zheng, Y., Fei, Z., Yu, J. Z., Isakeit, T., Wheeler, T., Frommer, W. B., He, P., Bogdanove, A. J., and Shan, L. 2017. TAL effector driven induction of a SWEET gene confers susceptibility to bacterial blight of cotton. Nat. Commun. 8:15588

Dekel, E., and Alon, U. 2005. Optimality and evolutionary tuning of the expression level of a protein. Nature 436:588-592.

Denny, T. 2006. Plant pathogenic Ralstonia species. Pages 573-644 in: Plant-Associated Bacteria. S. S. Gnanamanickam, ed. Springer, Dordrecht, The Netherlands.

Denny, T., and Hayward, A. C. 2001. Ralstonia. Pages 165-189 in: Laboratory Guide for Identification of Plant Pathogenic Bacteria, 3rd Ed., N. Schaad, J. B. Jones, and W. Chun, eds. APS Press, St. Paul, MN, U.S.A.

Dixon, G. R., and Pegg, G. F. 1972. Changes in amino-acid content of tomato xylem sap following infection with strains of Verticillium alboatrum. Ann. Bot. 36:147-154.

Driouich, A., Follet-Gueye, M. L., Vicré-Gibouin, M., and Hawes, M. 2013. Root border cells and secretions as critical elements in plant host defense. Curr. Opin. Plant Biol. 16:489-495.

Dulla, G., and Lindow, S. E. 2008. Quorum size of Pseudomonas syringae is small and dictated by water availability on the leaf surface. Proc. Natl. Acad. Sci. U.S.A. 105:3082-3087.

Elphinstone, J. G. 2005. The current bacterial wilt situation: a global overview. Pages 9-28 in: Bacterial Wilt Disease and the Ralstonia solanacearum Species Complex. C. Allen, P. Prior, and A. C. Hayward, eds. APS Press, St. Paul, MN, U.S.A.

Evert, R., and Eichhorn, S. 2006. Esau's Plant Anatomy: Meristems, Cells, and Tissues of the Plant Body: Their Structure, Function, and Development. John Wiley \& Sons, Inc., Hoboken, NJ, U.S.A.

Fatima, U. and Senthil-Kumar, M. 2015. Plant and pathogen nutrient acquisition strategies. Front. Plant Sci. 6:750.

Genin, S., and Denny, T. P. 2012. Pathogenomics of the Ralstonia solanacearum species complex. Annu. Rev. Phytopathol. 50:67-89.

Gonzales, E. T., and Allen, C. 2003. Characterization of a Ralstonia solanacearum operon required for polygalacturonate degradation and uptake of galacturonic acid. Mol. Plant-Microbe Interact. 16:536-544.

Gudelj, I., Beardmore, R. E., Arkin, S. S., and MacLean, R. C. 2007. Constraints on microbial metabolism drive evolutionary diversification in homogeneous environments. J. Evol. Biol. 20:1882-1889.

Hayward, A. C. 1991. Biology and epidemiology of bacterial wilt caused by pseudomonas solanacearum. Annu. Rev. Phytopathol. 29:65-87.

Heckman, K. L., and Pease, L. R. 2007. Gene splicing and mutagenesis by PCR-driven overlap extension. Nat. Protoc. 2:924-932.

Huang, Q., and Allen, C. 2000. Polygalacturonases are required for rapid colonization and full virulence of Ralstonia solanacearum on tomato plants. Mol. Plant Pathol. 57:77-83.

Jacobs, J. M., Babujee, L., Meng, F., Milling, A., and Allen, C. 2012. The in planta transcriptome of Ralstonia solanacearum: Conserved physiological and virulence strategies during bacterial wilt of tomato. MBio 3:e00114-12.

Kanehisa, M., and Goto, S. 2000. KEGG: Kyoto Encyclopedia of Genes and Genomes. Nucleic Acids Res. 28:27-30.

Khokhani, D., Lowe-Power, T., Tran, T., and Allen, C. 2017. A single regulator mediates strategic switching between attachment/spread and growth/virulence in the plant pathogen Ralstonia solanacearum. MBio 8:e00895-17.

Khokhani, D., Tran, T., Lowe-Power, T., and Allen, C. 2018. Plant assays for quantifying Ralstonia solanacearum virulence. Bio-protocols 8:18.

Knipfer, T., Cuneo, I. F., Brodersen, C. R., and McElrone, A. J. 2016. In situ visualization of the dynamics in xylem embolism formation and removal in the absence of root pressure: A study on excised grapevine stems. Plant Physiol. 171:1024-1036.
Kolbe, A., Tiessen, A., Schluepmann, H., Paul, M., Ulrich, S., and Geigenberger, P. 2005. Trehalose 6-phosphate regulates starch synthesis via posttranslational redox activation of ADP-glucose pyrophosphorylase. Proc. Natl. Acad. Sci. U.S.A. 102:11118-11123.

Korenblum, E., Dong, Y., Szymanski, J., Panda, S., Jozwiak, A., Massalha, H., Meir, S., Rogachev, I., and Aharoni, A. 2020. Rhizosphere microbiome mediates systemic root metabolite exudation by root-to-root signaling. Proc. Natl. Acad. Sci. U.S.A. 117:3874-3883.

Kravchenko, L. V., Azarova, T. S., Leonova-Erko, E. I., Shaposhnikov, A. I., Makarova, N. M., and Tichonovich, I. A. 2003. Root exudates of tomato plants and their effect on the growth and antifungal activity of Pseudomonas strains. Microbiology 72:37-41.

Liu, H., Zhang, S., Schell, M. A., and Denny, T. P. 2005. Pyramiding unmarked deletions in Ralstonia solanacearum shows that secreted proteins in addition to plant cell-wall-degrading enzymes contribute to virulence. Mol. Plant-Microbe Interact. 18:1296-1305.

Lowe, T. M., Ailloud, F., and Allen, C. 2015. Hydroxycinnamic acid degradation, a broadly conserved trait, protects Ralstonia solanacearum from chemical plant defenses and contributes to root colonization and virulence. Mol. Plant-Microbe Interact. 28:286-297.

Lowe-Power, T. M., Hendrich, C. G., von Roepenack-Lahaye, E., Li, B., Wu, D., Mitra, R., Dalsing, B. L., Ricca, P., Naidoo, J., Cook, D., Jancewicz, A., Masson, P., Thomma, B., Lahaye, T., Michael, A. J., and Allen, C. 2018a. Metabolomics of tomato xylem sap during bacterial wilt reveals Ralstonia solanacearum produces abundant putrescine, a metabolite that accelerates wilt disease. Environ. Microbiol. 20:1330-1349.

Lowe-Power, T. M., Jacobs, J. M., Ailloud, F., Fochs, B., Prior, P., and Allen, C. 2016. Degradation of the plant defense signal salicylic acid protects Ralstonia solanacearum from toxicity and enhances virulence on tobacco. MBio 7:e00656-16.

Lowe-Power, T. M., Khokhani, D., and Allen, C. 2018b. How Ralstonia solanacearum exploits and thrives in the flowing plant xylem environment. Trends Microbiol. 26:929-942.

Lunn, J. E., Delorge, I., Figueroa, C. M., Van Dijck, P., and Stitt, M. 2014. Trehalose metabolism in plants. Plant J. 79:544-567.

Macho, A. P., Zumaquero, A., Ortiz-Martín, I., and Beuzón, C. R. 2007. Competitive index in mixed infections: A sensitive and accurate assay for the genetic analysis of Pseudomonas syringae-plant interactions. Mol. Plant Pathol. 8:437-450.

McGarvey, J. A., Denny, T. P., and Schell, M. A. 1999. Spatial-temporal and quantitative analysis of growth and EPS I production by Ralstonia solanacearum in resistant and susceptible tomato cultivars. Phytopathology 89:1233-1239.

MacIntyre, A. M., Barth, J. X., Pellitteri Hahn, M. C., Scarlett, C. O., Génin, S., and Allen, C. 2020. Trehalose synthesis contributes to osmotic stress tolerance and virulence of the bacterial wilt pathogen Ralstonia solanacearum. Mol. Plant-Microbe Interact. 33:462-473.

Monteiro, F., Solé, M., van Dijk, I., and Valls, M. 2012. A chromosomal insertion toolbox for promoter probing, mutant complementation, and pathogenicity studies in Ralstonia solanacearum. Mol. Plant-Microbe Interact. 25:557-568.

Mori, Y., et al. 2017. Involvement of ralfuranones in the quorum sensing signalling pathway and virulence of Ralstonia solanacearum strain OE1-1. Mol. Plant Pathol. 19:454-463.

Nardini, A., Lo Gullo, M. A., and Salleo, S. 2011. Refilling embolized xylem conduits: Is it a matter of phloem unloading? Plant Sci. 180:604611.

Paul, M. J., Primavesi, L. F., Jhurreea, D., and Zhang, Y. 2008. Trehalose metabolism and signaling. Annu. Rev. Plant Biol. 59:417-441.

Pérez-Donoso, A. G., Greve, L. C., Walton, J. H., Shackel, K. A., and Labavitch, J. M. 2007. Xylella fastidiosa infection and ethylene exposure result in xylem and water movement disruption in grapevine shoots. Plant Physiol. 143:1024-1036.

Perrier, A., Barlet, X., Peyraud, R., Rengel, D., Guidot, A., and Genin, S. 2018. Comparative transcriptomic studies identify specific expression patterns of virulence factors under the control of the master regulator PhcA in the Ralstonia solanacearum species complex. Microb. Pathog. 116:273-278.

Peyraud, R., Cottret, L., Marmiesse, L., Gouzy, J., and Genin, S. 2016. A resource allocation trade-off between virulence and proliferation drives metabolic versatility in the plant pathogen Ralstonia solanacearum. PLoS Pathog. 12:e1005939.

Pfeiffer, T., and Bonhoeffer, S. 2004. Evolution of cross-feeding in microbial populations. Am. Nat. 163:E126-E135.

Pollock, C. J., and Farrar, J. 1996. Source-sink relations: The role of sucrose. Pages 261-279 in: Environmental Stress and Photosynthesis. N. R. Baker, ed. Springer, Dordrecht, The Netherlands. 
Polz, M. F., and Cordero, O. X. 2016. Bacterial evolution: Genomics of metabolic trade-offs. Nat. Microbiol. 1:16181.

Poueymiro, M., Cazalé, A. C., François, J. M., Parrou, J. L., Peeters, N., and Genin, S. 2014. A Ralstonia solanacearum type III effector directs the production of the plant signal metabolite trehalose-6-phosphate. MBio 5:e02065-14.

Prior, P., Ailloud, F., Dalsing, B. L., Remenant, B., Sanchez, B., and Allen, C. 2016. Genomic and proteomic evidence supporting the division of the plant pathogen Ralstonia solanacearum into three species. BMC Genomics 17:90.

Salanoubat, M., and Belliard, G. 1989. The steady-state level of potato sucrose synthase mRNA is dependent on wounding, anaerobiosis and sucrose concentration. Gene 84:181-185.

Siebrecht, S., Herdel, K., Schurr, U., and Tischner, R. 2003. Nutrient translocation in the xylem of poplar-Diurnal variations and spatial distribution along the shoot axis. Planta 217:783-793.

Sköt, L., and Egsgaard, H. 1984. Identification of inositol and O-methylscyllo-inositol in pea root nodules. Planta 161:32-36.

Sonnewald, U., and Willmitzer, L. 1992. Molecular approaches to sinksource interactions. Plant Physiol. 99:1267-1270.

Tans-Kersten, J., Guan, Y., and Allen, C. 1998. Ralstonia solanacearum pectin methylesterase is required for growth on methylated pectin but not for bacterial wilt virulence. Appl. Environ. Microbiol. 64:4918-4923.

Teixeira, A., Ribeiro, L., Rezende, S., Barros, E., and Moreira, M. 2012. Development of a method to quantify sucrose in soybean grains. Food Chem. 130:1134-1136.

Tran, T. M., MacIntyre, A., Hawes, M., and Allen, C. 2016. Escaping underground nets: Extracellular DNases degrade plant extracellular traps and contribute to virulence of the plant pathogenic bacterium Ralstonia solanacearum. PLoS Pathog. 12:e1005686.

Vasse, J. 1995. Microscopic studies of intercellular infection and protoxylem invasion of tomato roots by Pseudomonas solanacearum. Mol. Plant-Microbe Interact. 8:241-251.

Vílchez, J. I., Yang, Y., He, D., Zi, H., Peng, L., Lv, S., Kaushal, R., Wang, W., Huang, W., Liu, R., Lang, Z., Miki, D., Tang, K., Paré,
P. W., Song, C.-P., Zhu, J.-K., and Zhang, H. 2020. DNA demethylases are required for myo-inositol-mediated mutualism between plants and beneficial rhizobacteria. Nat. Plants 6:983-995.

Wadhams, G. H. and Armitage, J. P. 2004. Making sense of it all: Bacterial chemotaxis. Nat. Rev. Mol. Cell Biol. 5:1024-37.

Wang, E. L. and Bergeson, G. B. 1974. Biochemical changes in root exudate and xylem sap of tomato plants infected with Meloidogyne incognita. J. Nematol. 6:194-202.

Wei, Z., Yang, T., Friman, V.-P., Xu, Y., Shen, Q., and Jousset, A. 2015 Trophic network architecture of root-associated bacterial communities determines pathogen invasion and plant health. Nat. Commun. 6:8413.

White, M. C., Chaney, R. L., and Decker, A. M. 1981. Metal complexation in xylem fluid. III. Electrophoretic evidence. Plant Physiol. 67:311-315.

Wu, D., von Roepenack-Lahaye, E., Buntru, M., de Lange, O., Schandry, N., Pérez-Quintero, A. L., Weinberg, Z., Lowe-Power, T. M., Szurek, B., Michael, A. J., Allen, C., Schillberg, S., and Lahaye, T. 2019. A plant pathogen type III effector protein subverts translational regulation to boost host polyamine levels. Cell Host Microbe 26:638-649.e5.

Xian, L., Yu, G., Wei, Y., Li, Y., Xue, H., Morcillo, R., Rufian, J., and Macho, A. 2019. A bacterial effector protein hijacks plant metabolism to support pathogen nutrition. Cell Host Microbe 28:548-557.e7.

Yang, C. H., and Crowley, D. E. 2000. Rhizosphere microbial community structure in relation to root location and plant iron nutritional status. Appl. Environ. Microbiol. 66:345-351.

Yao, J., and Allen, C. 2007. The plant pathogen Ralstonia solanacearum needs aerotaxis for normal biofilm formation and interactions with its tomato host. J. Bacteriol. 189:6415-6424.

Zhalnina, K., Louie, K. B., Hao, Z., Mansoori, N., da Rocha, U. N., Shi, S., Cho, H., Karaoz, U., Loqué, D., Bowen, B. P., Firestone, M. K., Northen, T. R., and Brodie, E. L. 2018. Dynamic root exudate chemistry and microbial substrate preferences drive patterns in rhizosphere microbial community assembly. Nat. Microbiol. 3:470-480.

Zuluaga, A. P., Puigvert, M., and Valls, M. 2013. Novel plant inputs influencing Ralstonia solanacearum during infection. Front. Microbiol. 4:349. 